\title{
Design and Research of wireless sensor data acquisition system based on dynamic clamping force of hydraulic power chuck
}

\author{
Yueming Zhang ${ }^{1, a}$, Xunxun $\mathrm{Chu}^{2, \mathrm{~b}}$ \\ ${ }^{1}$ Beijing University of Technology, Beijing 100124, China; \\ ${ }^{2}$ Beijing University of Technology, Beijing 100124, China; \\ azhangym@bjut.edu.cn, ${ }^{b}$ chuxx@emails.bjut.edu.cn
}

Keywords: hydraulic power chuck, dynamic clamping force, error compensation, sensor, upper computer

\begin{abstract}
Hydraulic power chuck clamping force has been the main factor of the precision ultra-precision machining precision of modern CNC machine tools. To solve this problem, the designed wireless sensor system can achieve the real-time detection of chuck dynamic clamping force. The system can provide on-site real-time monitoring data signals and wireless data transmission, and the PC can accept and display real-time dynamic monitoring results and other functions.At the same time, the system has a temperature error compensation function to ensure a more accurate function curves of the relationship between clamping force and chuck speed, and provides important theoretical basis in improving the modern CNC machine tools spindle speed as well as optimizing design of hydraulic cylinders and hydraulic power.
\end{abstract}

\section{Introduction}

In recent years, with the development of 4.0 industrial revolution, the development of high-speed cutting and hard cutting technology put forward higher requirements to improve speed CNC machine tools[1]. But with the rapid progress of spindle drive technology, workpiece clamping device of hydraulic power chuck has hindered the improvement of CNC machine tool spindle speed. Meanwhile, the good clamping effect of hydraulic power chuck is the premise to achieve CNC machine tools precision ultra-precision machining. Hydraulic power chuck with high-speed rotation of the cylinder as the power source, when chuck at high speed, chuck jaws under high speed centrifugal force makes the chuck clamping force dropped substantially.Since the loss of clamping force is proportional to the square of speed. The loss of clamping force reaches a critical position will result in artifacts to fall off which will causing serious accidents[2]. At high speed, reasonable dynamic increase chuck clamping force can effectively avoid the accident, however, if the clamping force is too large and may cause damage and deformation of the workpiece.

In order to study the dynamic change trend of the chuck clamping force, the paper takes wedge-speed power chuck (KT54) and the supporting rotary cylinder (P22-200) of Huhhot Zhonghuan limited liability company as the research object, and has designed and developed a set of wireless sensor monitoring data acquisition system for textting hydraulic power chuck clamping force. Through experiments, the paper gained function curve equation of the clamping force loss with the increase of the speed of clamping force, which providing important technical support in the design and development and optimization of different functions chucks.

\section{The structure of the wireless sensor measuring device}

The overall structure of the wireless sensor. In order to facilitate stable clamping sensor, the sensor is designed circular shape. This kind of structure to achieve the advantages of small volume, convenient installation, reliable operation of the sensor. As shown in Fig. 1. 


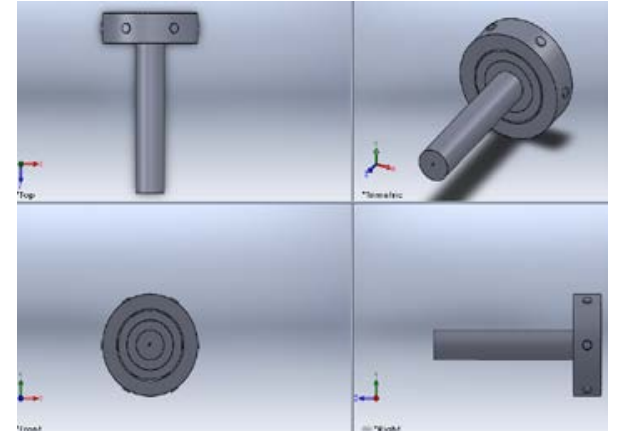

Fig. 1 The overall structure of the sensor

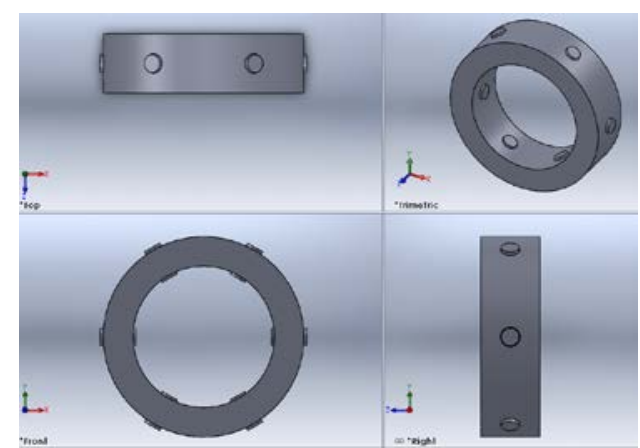

Fig. 2 The outer ring and pressure column

The outer ring structure. For different fixture types (chuck or calipers), different number of jaws(three or four jaws chuck), on the outer ring uniform six adjustable length compression column, so that it can guarantee the stability of collected data effectively, but also can measure the clamping force for clamping in different ways. The arrangement of pressure column is shown in Fig. 2.

The inner ring structure. The inner ring is the main force unit, to work under maximum load: $100 \mathrm{KN}$, to withstand the maximum rotational speed: $10000 \mathrm{r} / \mathrm{min}$. Inner ring of optimization design, through the establishment of mathematical analysis model using the powerful calculation function of Ansys to calculate the relationship between the clamping force and the inner ring strain.Through the optimization analysis, the optimal ratio of diameter is: $0.679 \approx 2 / 3$.

The arrangement of the strain gauge. Data acquisition sensor patch on the inner ring are arranged uniformly. In a different position decorate a strain gauge,the strain can be more accurately displayed. Each point represented by a combination of two strain gauges, so that it can eliminate measurement error in the case of the unevenness of the biasing force. Its layout is shown in Fig. 3.

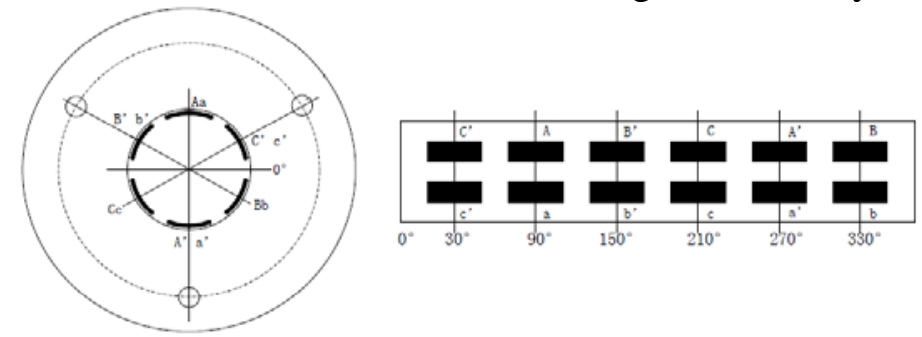

Fig. 3 The arrangement of the strain gauge

Handle construction of the sensor. Handle sensor is designed in a cylindrical shape, the sensor circuit board is arranged in the hands, the design can not only reduce the weight of the equipment, but also ensures that the sensor body stiffness requirements.

\section{The data acquisition of the wireless sensor measuring device}

MCU temperature error compensation[3] scheme. Setting the stress scale factor of 1 (setting input is 10000), and setting stress offset coefficient is 0 . Gradually raising the temperature from room temperature, recording the corresponding display values: temperature T, jaw measured value V3 (or four claw measurements V4). The collected data is recorded to EXCEL. The discrete points are engaged to the linear equation, then the scale factor is the error compensation parameters. Then setting the parameter to EEPROM.

Sensor master control module. The main control module uses C8051F500 microcontroller as the MCU. MCU circuit diagram shown in Fig. 4. 


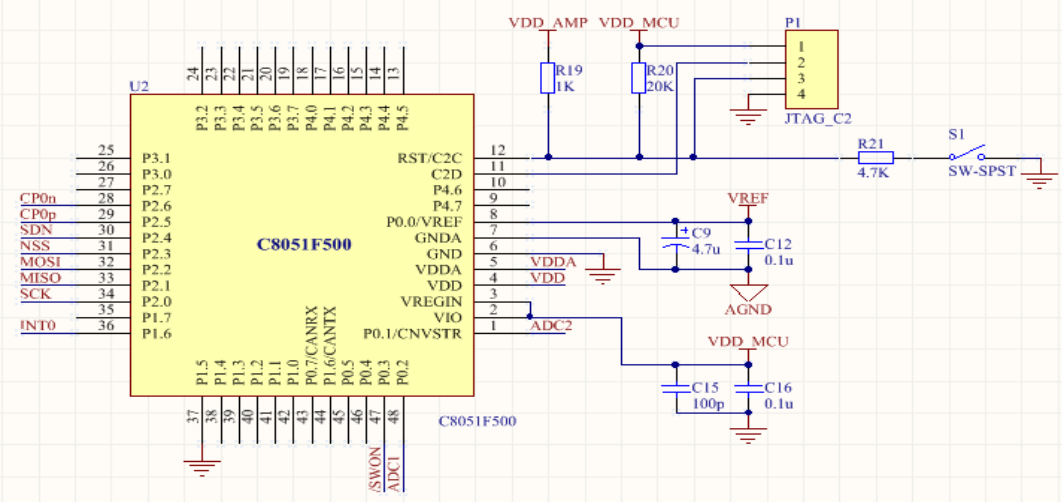

Fig. 4 MCU circuit diagram

Wireless communication transceiver module. Wireless communication transceiver module uses a 20-pin QFN packaged chip Si4431. Si4431 chip is a wireless transceiver chip of highly integrated, low-power, multi-frequency EZRadioPRO series, internal integrated power amplifier, wake-up timer, digital modem and receive data FIFO and configurable GPIO, etc. Si4431 is widely used as a wireless communication module. It can be applied to UAS control, robot control and spatial data networks. Wireless transceiver circuit diagram wiring diagram shown in Fig. 5 and Fig. 6.

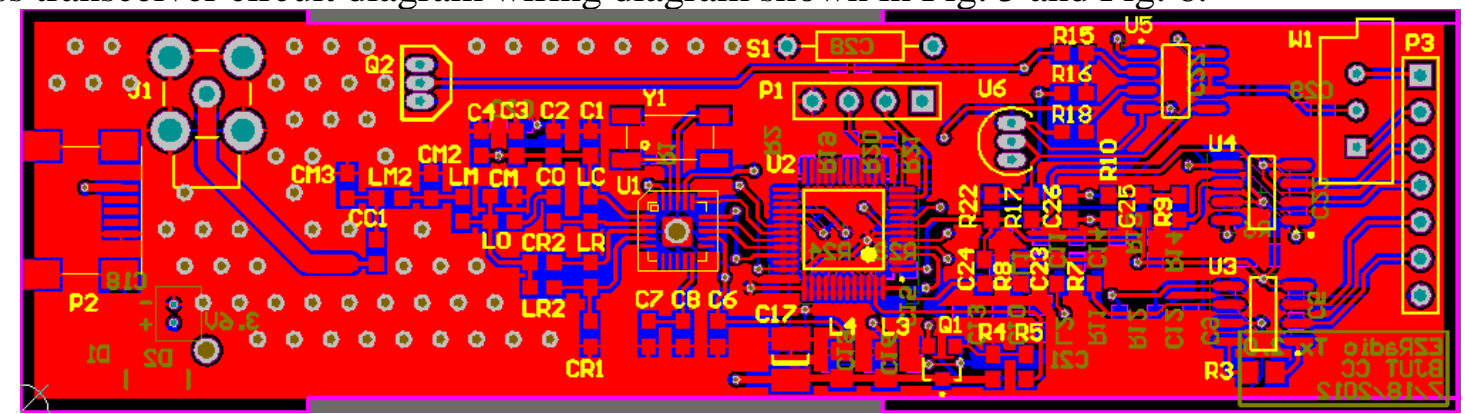

Fig. 5 Total wireless receiver circuit wiring diagram

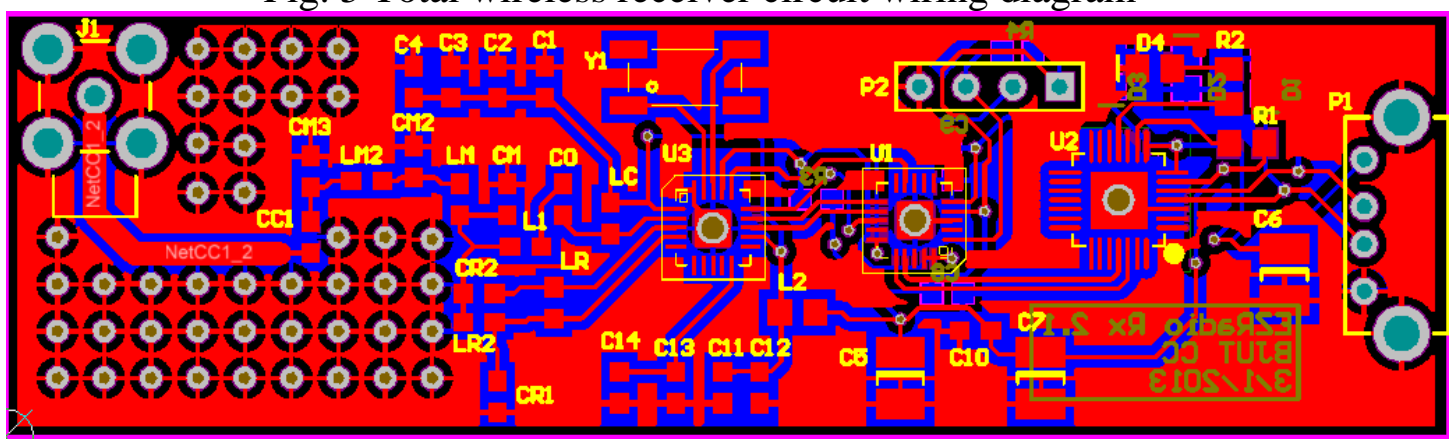

Fig. 6 Total wireless transmitter circuit wiring diagram

\section{The data receiving and analysis of wireless sensor measuring device}

Setting up software testing parameters: selecting the option $\mathrm{X}$-axis and $\mathrm{Y}$-axis, setting up equipment using COM port. Testing run the equipment to make sure the data collected is correct. First, the software generates error compensation value based on the collected data, then to set the error compensation. Wireless sensor devices sends data to the PC software. 


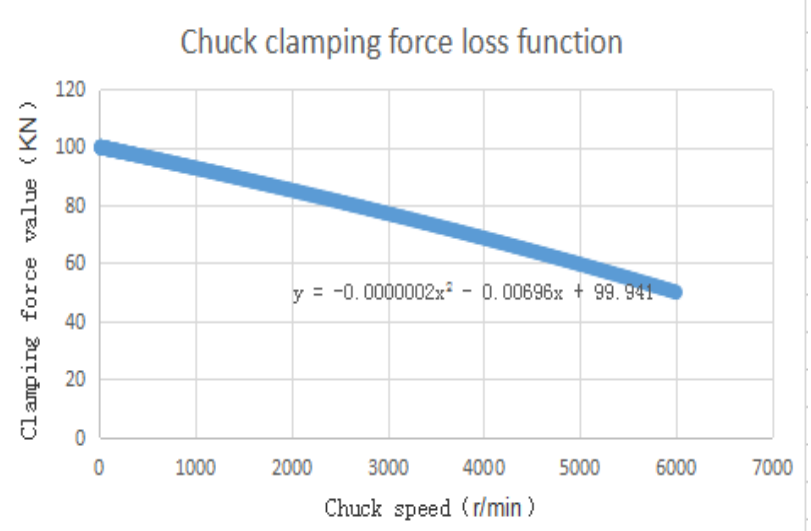

Fig. 7 Hydraulic power chuck clamping force loss diagra

Analysis of test results: Using least squares regression curve fitting method to get the fitting curve. We can get the functional relationship that the loss of clamping force is proportional to the square of speed. As shown in Fig. 7.

\section{Summary}

For a long time, the research of high speed power chuck is less in our country. Making China's precision ultra-precision CNC machine tool manufacturing technology is far behind international standards. With the rapid development of industry 4.0, machine spindle speed is gradually increasing, the clamping force of high-speed movement chuck part becomes a pressing problem. The wireless sensor data acquisition system can keep stable working state under the condition of high speed running and it can stably and accurately real-time measure data and get accurate clamping force loss function. The equipment makes up for the defects of high speed rotating chuck clamping force detection, it also can shorten the development cycles of chuck and provide some technical support.

\section{Acknowledgments}

This work was financially supported by "the National Science and Technology Major Project (2012ZX04002061)”.

\section{References}

[1] Rambod R,Sali A M. Multiple impedence control of space free-flying robots via virtual linkages[J]. Acta Astronautica, 2010, 66(5): 748-759.

[2] G.J. Javier, B. Eduardo, Kinematic and dynamic simulaton of multibody systems: the real-time challenge, New York, Springer-Verlag, 1993.

[3] S. Joseph, High Speed Power Chuck, US, Patent 4,097,053(1978). 